\title{
Paradigmas psiquiátricos y psicofarmacología. ¿Relevancia del órgano-dinamismo de Henry Ey?
}

\section{Psychiatric paradigms and psychopharmacology}

\section{Paradigmas psiquiátricos e psicofarmacologia. Relevância da organo-dinâmica de Henry Ey?}

Mauricio Toledo

ORCID ID: 0000-0001-9055-7432

Unidad Farmacología Clínica, Hospital Vilardebó, Uruguay

Ramiro Almada

ORCID ID: 0000-0002-3012-6443

Hospital Vilardebó, Uruguay

Luis Villalba

ORCID ID: 0000-0001-6337-4913

Asociación Psicoanalítica del Uruguay, Uruguay

Autor referente: mauricio.toledo1@gmail.com

Historia Editorial:

Recibido: 29/06/2018

Aceptado: 24/10/2018

\section{RESUMEN}

La psicofarmacología se ha constituido a lo largo de las últimas décadas en una herramienta fundamental para el abordaje del tratamiento de los trastornos psiquiátricos. Como todos los desarrollos tecnológicos eficaces y capaces de generar cambios sustantivos tiene luces y sombras. El desarrollo de los antipsicóticos, antidepresivos y ansiolíticos permitió comenzar a tratar eficazmente los

procesos mórbidos psiquiátricos como nunca antes se había realizado. A tal punto, que socavó el concepto de asilo psiquiátrico por el de hospital o unidades de atención, es decir, se generó una nueva organización asistencial. Sin embargo, aún estamos lejos de una opción fármacoterapéutica óptima. En un contexto asistencial donde se observa un incremento del consumo de 
psicofármacos, el desarrollo por parte de la industria farmacéutica de moléculas que no constituyen novedades terapéuticas y un sistema de investigación tutelado por la mirada de la medicina basada en las evidencias. A su vez, la psiquiatría actual está inmersa en un pluralismo teórico y epistemológico que ha desterrado a la psicopatología como forma de entender o interpretar el síntoma. Esta doble condición de pluralismo teórico y un uso a veces inadecuado de la fármaco-terapia condicionan nuestra práctica clínica. En el presente trabajo tenemos como objetivo abordar genéricamente el desarrollo de la psiquiatría desde la perspectiva de los paradigmas, la génesis y el desarrollo psicofarmacológico y considerar la relevancia de un modelo psicopatológico como el organodinamismo de Henry Ey.

Palabras clave: Psiquiatría; Paradigmas; Psicofarmacología; Órgano-dinamismo

\section{ABSTRACT}

Psychopharmacology has been constituted over the last decades in a fundamental tool for addressing the treatment of psychiatric disorders. Like all technological developments that are effective and capable of generating substantive changes, there are lights and shadows. The development of antipsychotics, antidepressants and anxiolytics allowed to begin to effectively treat morbid psychiatric processes as never before. To such an extent, that it undermined the concept of psychiatric asylum by that of hospital or care units, that is, a new healthcare organization was created. However, we are still far from an optimal pharmacotherapeutic option. In an assistance context where there is an increase in the consumption of psychotropic drugs, the development by the pharmaceutical industry of molecules that do not constitute therapeutic novelties and a research system guided by the eyes of medicine based on evidence. In turn, current psychiatry is immersed in a theoretical and epistemological pluralism that has banished psychopathology as a way of understanding or interpreting the symptom. This double condition of theoretical pluralism and sometimes inadequate use of the drug-therapy condition our clinical practice. In this work we aim to address generically the development of psychiatry from the perspective of paradigms, genesis and psychopharmacological development and consider the relevance of a psychopathological model such as Henry Ey's organo-dynamism.

Keywords: Psychiatry; Paradigms; Psychopharmacology; Organo-dynamism

\section{RESUMO}

A psicofarmacologia tem se constituído nas últimas décadas em uma ferramenta fundamental para abordar o tratamento de transtornos psiquiátricos. Como todos os desenvolvimentos tecnológicos que são eficazes e capazes de gerar mudanças substantivas, há luzes e sombras. O desenvolvimento de antipsicóticos, antidepressivos e ansiolíticos permitiu começar a tratar eficazmente os processos psiquiátricos mórbidos como nunca antes. De tal forma, que minou o conceito de asilo psiquiátrico por meio de unidades hospitalares ou de atendimento, ou seja, uma nova organização de saúde foi criada. No entanto, ainda estamos longe de ser uma ótima opção farmacoterapêutica. Em um contexto de assistência onde 
há um aumento no consumo de psicofármacos, o desenvolvimento pela indústria farmacêutica de moléculas que não constituem novidades terapêuticas e um sistema de pesquisa guiado pelos olhos da medicina baseada em evidências. Por sua vez, a psiquiatria atual está imersa em um pluralismo teórico e epistemológico que baniu a psicopatologia como uma maneira de entender ou interpretar o sintoma. Essa dupla condição de pluralismo teórico e, às vezes, uso inadequado da farmacoterapia condiciona nossa prática clínica. Neste trabalho pretendemos abordar genericamente o desenvolvimento da psiquiatria na perspectiva de paradigmas, gênese e desenvolvimento psicofarmacológico e considerar a relevância de um modelo psicopatológico como o organodinamismo de Henry Ey.

Palavras-chave: Psiquiatria; Paradigmas; Psicofarmacologia; Organo-dinâmica

\section{¿Por qué una historia en la comprensión epistemológica del campo} psiquiátrico?

a psiquiatría es una disciplina médica compleja puesto que, como en ninguna

otra rama de la medicina, el estudio de la enfermedad mental se desarrolla tanto en el campo de las disciplinas científico-naturales como en el de las disciplinas humanas del conocimiento. Debido a esta intersección, Berrios (2011) denomina a la psiquiatría como una disciplina híbrida. Es decir, una disciplina que se da en esa intersección de lo natural y cultural. Esta apreciación no es menor, ya que las dificultades epistemológicas de la disciplina surgen justamente debido al carácter híbrido de la misma. Lanteri Laura (2000) sugiere que la psiquiatría sólo puede estudiarse distinguiendo epistemologías regionales, es decir "sin que ningún metalenguaje pueda ser capaz de unificarlas" (p. 303). Sin embargo, más allá de las dificultades epistemológicas, la realidad clínica del sufrimiento psíquico, que es propio del campo antropológico, esta descrita desde la antigüedad.

En el campo de la psiquiatría los términos, conceptos y teorías se entrelazan, fluctúan y contextualizan en un sentido histórico que a su vez es discontinuo. El conocimiento histórico en psiquiatría no es entonces un conocimiento poco trascendente que dé una erudición decorativa a su enunciado. El conocimiento histórico contextualiza e informa sobre el alcance y el significado de los conceptos. Berrios (2011) considera que en la 
evaluación de un concepto desde el punto de vista epistemológico debe haber información tanto sobre su construcción histórica como sobre su estructura conceptual. A su vez, Lanteri Laura (2000) se refiere a que:

no puede considerarse ni una exploración psiquiátrica ni una discusión diagnóstica sin la presencia en segundo plano de ciertos aspectos de la historia de la psiquiatría en tanto que estos aspectos han constituido el contenido del thesaurus semioticus que se ha ido constituyendo acumulativamente. (p. 28) Lanteri Laura (2000) utiliza el criterio paradigmático como criterio de periodización de la historia de la psiquiatría. El mismo intenta adaptar el concepto de paradigma desarrollado por Thomas S. Kuhn. Lanteri Laura define de esta manera el concepto de paradigma utilizado en su análisis:

...concepto bastante global que, durante un cierto período, servirá para regular toda una serie de conocimientos teóricos y prácticos en uso, no como una concepción teórica global de la que aquellos provendrían sino como la delimitación y organización de cierto campo de acción en el que diferentes posibilidades se complementarían u opondrían. Lo que unifica (durante cierto tiempo) esa serie de representaciones teóricas y prácticas que se acomodan y excluyen entre sí. (p. 53)

El criterio de paradigma de Lanteri Laura no ha sido, desde luego, aceptado unánimemente. Allí tenemos la crítica que le realiza Berrios (2008) en la historia de los síntomas de los trastornos mentales, o la que escribe Barcia Salorio (2012).

El hecho es que en psiquiatría no ha aparecido nunca un paradigma en el sentido de Kuhn que haya asumido los anteriores, tal como ha ocurrido en otras ciencias, por lo que en todo momento de modo más o menos visible conviven una multitud de modelos. Seguramente sería más comprensible el análisis de la historia de la psiquiatría de un modo sincrónico que diacrónico, es 
decir, analizar la evolución de determinados planteamientos conceptuales más que atender la evolución temporal. (, p. 1)

Más allá de su oposición al criterio paradigmático, Barcia Salorio (2012) asume una posición discontinuista:

Por razones extracientíficas unos planteamientos se han impuesto a otros, respecto a la psiquiatría que podemos llamar "oficial", pero estos no desaparecen y están agazapados hasta que en otro momento histórico, también por razones no científicas, vuelven al primer plano. Por ello en cada período histórico domina una tendencia que se impone a las demás no porque sea mejor, en el sentido de que explique más hechos de la enfermedad mental, sino porque los dirigentes más significativos de esa orientación tienen mayor influencia debido a otras circunstancias. ( p. 2)

Un enfoque como el paradigmático a menudo es visto como anti-científico, sin embargo, como dice Levin (2010):

la ciencia así concebida no pierde valor como generadora de conocimiento capaz de motorizar el progreso de la civilización humana. Por el contrario, permite situar a la producción científica en un período histórico, determinada por los múltiples atravesamientos que experimenta: políticos, sociales, culturales. (p. 7)

Lanteri Laura (2000) describe 3 periodos en su análisis, caracterizados por la presencia de un paradigma: 1) Paradigma de la Alienación Mental (hipótesis de la afección mental única, lo que da unidad al período) que se desarrolla aproximadamente entre finales del S. XVIII y mediados del S. XIX. 2) Paradigma de las Enfermedades Mentales (distintas E.M. irreductibles entre sí) que transcurre hasta los inicios del S. XX. 3) Paradigma las de las Grandes Estructuras Psicopatológicas transcurre hasta finales de la década de los 70. 


\section{¿Dónde estamos?}

¿Cuál es el estado actual de la Psiquiatría desde el punto de vista de sus paradigmas? Algunas posiciones sostienen que el pluralismo tanto teórico como epistemológico parece definir mejor nuestra época. Kendler (2016) se pregunta en uno de sus trabajos ¿Qué clase de fenómeno es un trastorno psiquiátrico? Considera que en psiquiatría hay tres perspectivas filosóficas fundamentales: el realismo, el pragmatismo y el constructivismo. Kendler los describe de la siguiente manera.

El realismo presupone que el contenido de la ciencia es real e independiente de las actividades humanas. Aunque distingue dos diferentes "sabores", uno basado en la química, cuyo ejemplo paradigmático son los elementos de la tabla periódica, y otro basado en la biología, en que el paradigma es la "especie". Entiende que este último se adapta mucho mejor a la psiquiatría.

El enfoque pragmático de los trastornos psiquiátricos sólo busca categorías que funcionen bien en el mundo. No tiene ningún postulado sobre la realidad de estos trastornos. Resulta problemático ya que a diferencia de otras ramas de la medicina nos obliga a defender la realidad de los trastornos que tratamos.

El constructivismo se ha relacionado con activistas anti-psiquiatría, pero debemos admitir que las fuerzas sociales desempeñan un rol en la creación de nuestros diagnósticos, lo mismo que en muchas ciencias. Sin embargo, los trastornos psiquiátricos socialmente construidos son infrecuentes.

\section{¿Un paradigma actual?}

Puede ser visto el periodo actual como un largo periodo de crisis paradigmática. Stagnaro (2006) sintetiza la situación actual:

El paradigma dominante que nos contenía hasta los años ' 70 , el de las Grandes Estructuras Psicopatológicas, surgido de la influencia de la Gestaltheorie, la lingüística estructural y la neurología globalista y expresado en las corrientes fenomenológicas, especialmente en la obra de Minkowsky y 
Binswanger, en el psicoanálisis y en el organodinamismo de Henri Ey, ha sufrido una desagregación tal, que bien podemos catalogar la situación actual como de crisis paradigmática, en el sentido en que emplea este término Thomas Kuhn. (p. 190)

A lo que añade:

En efecto, a poco de echar una mirada crítica sobre las prácticas y teorías de la psiquiatría actual, debemos reconocer que estamos progresivamente obligados a aceptar relaciones cada vez más arbitrarias entre nuestros gestos técnicos y nuestra capacidad de formalizarlos teóricamente en forma consensuada. ( $p$. 190)

Dentro de esta carencia de un paradigma dominante, o en el concierto de un panorama de complejidad y multiplicidad, sin embargo puede vislumbrarse una tendencia:

El análisis específico del campo de la psiquiatría, dentro de las especialidades médicas a las cuales pertenece, nos permite verificar que el reduccionismo biomédico penetró con fuerza, a lo largo de las últimas tres décadas, en el ámbito de la medicina mental. En el contexto actual de "crisis paradigmática" se dibuja una aproximación paradigmática prevaleciente caracterizada por:

a. Identificación objetiva y categorial de los trastornos mentales por vía de una descripción "a-teórica”, como propone el Diagnostic Statical Mental (DSM) IV; b. Progresiva correlación biunívoca entre cada trastorno y su fisiopatología cerebral;

c. Correlación entre dicha fisiopatología y su corrección farmacológica, combinada con psicoterapia cognitivo-comportamental. (Levin, 2010, p. 271)

EI DSM ha tenido un rol primordial de gran influencia no sólo sobre la nosografía sino sobre distintos aspectos de la consideración conceptual de la patología mental. El fuerte posicionamiento de esta visión conceptual se ha acompañado de un 
debilitamiento correspondiente del análisis psicopatológico estructural, y en particular de la fenomenología. Largamente analizado, la más famosa referencia al respecto proviene sin embargo desde las propias entrañas de la Asociación de Psiquiatría Americana (APA, 2002), con el artículo de Nancy Andreasen (2007). En el artículo se señala, entre otras, como "consecuencias no deseadas" del DSM:

Los DSM se convirtieron sin querer en la autoridad máxima en psicopatología y nosología, universal y acríticamente aceptada. Se convirtieron también en la base para la enseñanza de la disciplina en todo Estados Unidos de América (EUA). Concordantemente los clásicos han sido olímpicamente ignorados. ( $p$. 111)

Determinando también, entre otras cosas que "la validez (de las categorías) ha sido sacrificada en nombre de la confiabilidad (reproductibilidad)" (p. 111). Para concluir que "En la actualidad el declive en la consideración de las complejidades psicopatológicas es tan severo, que puede ser referido como la muerte de la fenomenología en EUA" ( $p$. 108).

El DSM con su pretensión de a-teoricismo respecto a la etiología abandona el concepto de enfermedad y propone el de trastorno (disorder). Luego de dar una definición "pragmática" de trastorno mental admite que "no existe una definición que especifique adecuadamente los límites del concepto "trastorno mental", y que "la definición finalmente tomada es la misma que está desde el DSM III, y es tan útil como cualquier otra" (APA, 2002, p. XXIX).

Los problemas para una adecuada conceptualización no quedan limitados al DSM. Bajo un posicionamiento naturalista que considera a la enfermedad mental como reducible a una enfermedad cerebral se han realizado esfuerzos por desarrollar definiciones "naturalísticas" de la EM. En este contexto se enmarca la noción de “disfunción perjudicial” (Wakefield, 1992) y el concepto desarrollado por Boorse (1975) bajo su "teoría bioestadística". En ningún caso esas definiciones pudieron cumplir su 
objetivo de delimitar "categorías naturales objetivas" (Kingma, 2013). En la misma dirección, el Instituto Nacional de Salud Mental estadounidense, en su búsqueda de intentar establecer un vínculo directo entre las manifestaciones mentales y el substrato orgánico, ha abandonado el concepto de trastorno mental del DSM y desarrollado los Research Domain Criteria (Rdoc), abandonando la identificación de síndromes globales en privilegio de entidades que se acercan al tipo de los endofenotipos o fenotipos intermedios (Cuthbert, 2014). Por otra parte y desde posiciones distintas, ha tomado un fuerte posicionamiento el concepto de Salud Mental (Bertolote, 2008) vinculado al concepto de bienestar, remarcando la necesidad de considerar a este y no sólo al sufrimiento mental, y enmarcado en la consideración dimensional del problema (el continuo salud-enfermedad). Últimamente se han multiplicado también los movimientos en la sociedad que se oponen al uso del término "enfermedad" pues lo consideran "discriminatorio", judicativo, y eventualmente de por sí "estigmatizante". Estos movimientos han logrado una importante repercusión en el ámbito público con respuestas concretas en términos legislativos y de acciones gubernamentales.

Este complejo panorama donde coexisten y eventualmente compiten múltiples posiciones teóricas y conceptuales implica también un retroceso en la conceptualización psicopatológica de la enfermedad mental. El campo de la psiquiatría parece haberse desprendido o apartado de ese concepto que cumplió un rol nucleador para la psiquiatría hasta entrado el último cuarto del $S$ XX. Esta complejidad y heterogeneidad actual determina un aumento del abanico de situaciones de las que se ocupa la psiquiatría. De la siguiente manera se refería Lanteri Laura (2000) a este fenómeno:

si la legitimidad del núcleo central de la psiquiatría sigue siendo muy contestada, su periferia no deja de crecer y se nos exige que nos ocupemos de la orientación escolar, de las dificultades conyugales, de los problemas de la 
adopción y de muchos otros asuntos en los cuales nuestra competencia efectiva nos ha parecido siempre mal fundamentada. (p. 269)

El trabajo de Mc Hugh y Slavney (1985) constituye un ejemplo de intentar reconocer y admitir la complejidad y variedad de situaciones que terminan siendo comprendidas y constituyendo un expandido campo de la psiquiatría. Refiriendo el carácter, amorfo y agobiado por disputas de la psiquiatría contemporánea, los autores intentan forjar una estructura conceptual de la que puedan surgir una mejor investigación y una práctica moderna y coherente. Se refieren a "perspectivas" distintas como métodos de razonamiento estándar que están implícitos en el pensamiento psiquiátrico contemporáneo y deben hacerse explícitos: la perspectiva de la enfermedad, la dimensional, la de la conducta, y la de la historia vital. Cada una de estas perspectivas tiene su propio concepto de psicopatología, de lo que es normal y anormal en la vida mental.

\section{Psicofármacos: un poco de historia}

El desarrollo de la psicofarmacología tal como la conocemos en la actualidad tiene su inicio a mediados del siglo XX. Obviamente esta es producto de la ciencia de la época, la cual estaba impregnada del desarrollo tecnológico de posguerra. La emergencia de la psicofarmacología está vinculada a la síntesis de miles de moléculas que sustentaban a la química como paradigma terapéutico. El descubrimiento de estas moléculas psicotrópicas fue producto de la serendipia (serendipty) es decir, un descubrimiento inesperado o casual. Su impacto en el campo psiquiátrico fue sustantivo, ya que permitió generar otro curso evolutivo de los procesos mórbidos psiquiátricos. Si bien ya existían medicamentos con capacidades hipnóticas y ansiolíticas, uno de los aspectos singulares descubiertos fueron las propiedades antialucinatorias y antidelirantes así como antidepresivas. 
Según Healy (2002) la historia moderna de los fármacos y la "locura" comienza con la década de 1950 con la síntesis de la clorpromazina. Sin embargo, la travesía psicofarmacológica comienza en el siglo XIX con la síntesis de las moléculas orgánicas como la urea. Esto genera un cambio paradigmático en la química. En 1876 H. Caro, trabajando en Badische Analin und Soda Fabrick (BASF) sintetiza el azul de metileno. Por otro lado, C. Lauth en Inglaterra con un proceso similar sintetiza el Lauth's violet. Para entender la estructura y así poder patentar el producto, Caro le solicita al químico de Heidelberg A. Bernthsen estudiar las estructuras químicas. Bernthsen encuentra que ambas tienen el mismo núcleo pero distintas cadenas laterales, al cual denomina núcleo fenotiazinico. El azul de metileno tendrá un rol significativo en el desarrollo de las ciencias biológicas (Koch y la tinción mycobacterium y Ehrlich para el tratamiento de la malaria) a su vez, es el ancestro directo de la clorpromazina. Es de destacar que tanto los antidepresivos como los antipsicóticos derivan de los antihistamínicos (Healy, 2002).

\section{Síntesis de la clorpromazina: neurolépticos o antipsicóticos}

Si bien ya en 1950 P. Guiraud destaca los usos beneficiosos de la prometazina en la esquizofrenia y estados de agitación, se destaca su uso debido a las propiedades sedativas y este es el interés principal del desarrollo de estos antihistamínicos por el fabricante. Henri Laborit, cirujano militar, le sugiere a Rhone-Poulenc la posibilidad de optimizar los efectos estabilizantes a nivel del sistema nervioso central para el cocktail lítico (la hipótesis del cocktail lítico era evitar el stress inducido al organismo frente a los estados de hipotermia quirúrgica). Charpentier desarrolla una serie de nuevos antihistamínicos, siendo uno de esos compuestos el RP 4560 o clorpromazina. El amplio rango de acciones evidenciadas por este fármaco determina su nombre comercial en Europa, Largactil. En 1952 Pierre Deniker psiquiatra del Hospital SainteAnne, bajo la dirección de Jean Delay comienza con un plan inicial de utilización de hibernación artificial de Laborit. Deniker y Delay fueron los primeros en descubrir los 
efectos de la clorpromazina sin los otros elementos del cocktail. Estos autores fueron los primeros en demostrar los efectos psicotrópicos y otros grupos de trabajo que se conformaron en torno a la clorpromazina en Lyon y en Basilea confirman estos efectos (Healy, 2002).

En Basilea, Steck y Staenhelin utilizaron dosis más altas de clorpromazina que las del grupo de Paris. Siendo efectivas como antidelirantes y antialucinatorias, sin embargo devenían parkinsonianos. Debido a este fenómeno Steck propone que la clorpromazina actúa a nivel del cerebro medio motor y esto explica la acción beneficiosa. Se propusieron diferentes nombres a este grupo de sustancias como catárticos, neuroplégicos, ganglioplégicos y tranquilizantes. En 1957, durante el II Congreso Mundial de Psiquiatría en Zúrich, se discute acerca de la denominación de este grupo y finalmente queda zanjada con la propuesta de Delay como neurolépticos. Sin embrago, los estadounidenses manejan el termino de tranquilizantes mayores y luego adoptan el término antipsicóticos (Healy, 2002).

\section{Uso actual de psicofármacos}

En los últimos años se ha evidenciado un aumento del consumo de psicofármacos, la utilización de los antipsicóticos se ha incrementado a expensas de los antipsicóticos atípicos. Hálfdánarson et al. (2017) realizaron un trabajo en varios países tanto de la Unión Europea como de América y Asia. El periodo de estudio fue de 2004 a 2014. Si bien encontraron asimetrías en la tendencia del uso de diferentes antipsicóticos, hay un aumento en el uso de los antipsicóticos y es a predominio del uso de los atípicos. En España, la Agencia Española de Medicamentos y Productos Sanitarios (2007) llevo a cabo un trabajo de consumo de antipsicóticos durante el periodo comprendido entre 1992 y 2006 demostrando que el consumo de antipsicóticos se ha casi triplicado, principalmente debido al uso de los antipsicóticos atípicos. A su vez un trabajo canadiense realizado por Pringsheim y Gardner (2014) demuestra un aumento en la tendencia prescriptiva de antipsicóticos atípicos entre 2005 y 2012, destacándose el 
uso de la quetiapina. A su vez, los antidepresivos cumplen con este mismo fenómeno. Por ejemplo, en España la Agencia Española de Medicamentos y Productos Sanitarios (2015) demuestra un incremento del consumo de antidepresivos en el periodo 20002013, este ha sido de un $200 \%$ vinculado principalmente por el incremento de los inhibidores de recaptación de serotonina (ISRS). En Canadá, Morkem, Barber, Williamson \& Patten (2012) en un estudio efectuado en el primer nivel de atención observaron que la prevalencia de la prescripción de antidepresivos se ha incrementado desde el 2006 al 2012. A su vez, la tendencia en la prescripción de antidepresivos en Inglaterra durante el periodo 1998-2010 aumento en un promedio de $10 \%$ anual (llyas \& Moncrieff, 2012).

A nivel nacional, la Sexta Encuesta Nacional en Hogares sobre consumo de drogas, detecto que un $10,6 \%$ de la población considerada los consumió alguna vez en la vida y un $5 \%$ en los últimos 12 meses. A nivel hospitalario, un trabajo sobre utilización de antidepresivos realizado en el Hospital Policial Teixeira, Imbriago y Sarries (2015) detectó un incremento del consumo del 8\% entre el periodo 2010-2014.

Por otro lado, la polifarmacia (uso de dos o más medicamentos de la misma clase química o mismas características farmacológicas) es muy frecuente con el uso de esta clase de medicamentos. La prevalencia de polifarmacia es variable y oscila entre un 10 y 73\% dependiendo de la región y población estudiada (Gallego, Bonetti, Zhang, Kane \& Correll, 2012). Esta aumenta el riesgo de efectos adversos, interacciones farmacológicas (farmacocinéticas y farmacodinámicas), falta de cumplimiento terapéutico, errores de medicación y aumento de los costos.

\section{Buceando entre las evidencias}

Tradicionalmente, el beneficio de un medicamento o intervención terapéutica se evaluaba empíricamente en base a la respuesta clínica observable después de una intervención basada en el razonamiento fisiopatológico, el sentido común y la experiencia personal. En ese contexto epistemológico se inició el desarrollo de la 
psicofarmacología. Actualmente estamos bajo el paradigma de la Medicina Basada en Evidencia (MBE). Bajo este paradigma el concepto de beneficio se discrimina: la eficacia es el beneficio obtenido de un tratamiento en el ámbito de experimentación, la efectividad es aquel beneficio obtenido en la práctica clínica diaria, es decir en la realidad clínica y la eficiencia establece la relación económica de la efectividad de un tratamiento (Baños \& Farré, 2002). La MBE surge en la década de los años 90 del siglo XX en la Universidad McMaster de Canadá, se acuñó el término para designar a una nueva forma de práctica médica que agrega el manejo de la mejor evidencia disponible proveniente de la investigación, categorizada en distintos niveles en función de la confiabilidad que ofrecen diferentes herramientas metodológicas de análisis a la observación clínica tradicional. La fuerza del nivel de evidencia está determinada por el método-diseño de la evaluación de la intervención clínica, siendo el Ensayo Clínico Controlado (ECC) junto al Meta-análisis las herramientas metodológicas que mayor nivel de evidencia aportan (Evidence-Based Medicine Working Group, 1992). Sin embargo, el beneficio de un fármaco o medicamento (eficacia-evidencia) no necesariamente significa que el mismo tenga un claro beneficio en la práctica clínica diaria (efectividad), ya que en muchas ocasiones sobrepasar el umbral de la significación estadística no alcanza para tener una respuesta clínica adecuada. En psicofarmacología muchas veces puede ser difícil de obtener ya que el tamaño del efecto puede ser modesto, sobre todo a corto plazo. La psiquiatría no ha sido ajena al movimiento (MBE) fenómeno por lo cual se ha desarrollado el concepto de Psiquiatría Basada en la Evidencia (PBE). Sin embargo, desde hace más de diez años han surgido críticas y objeciones a la MBE, tanto desde sus aspectos metodológicos como epistemológicos.

\section{Alcances y limitaciones metodológicas}

Un primer aspecto a considerar, es la dificultad en transferir la información obtenida de los ECC o meta-análisis a la práctica clínica diaria (PCD). Varios factores podrían 
explicar este hiato (gap) o dificultad en la transferencia. Los ECC pueden clasificarse en explicativos y pragmáticos Baños y Farré (2002), los de tipo explicativos tienen el objetivo de evaluar la eficacia, y en ellos se controlan bien los sesgos y los factores de confusión. Los grupos son homogéneos, la diferencia del efecto se maximiza y en general se utilizan en las fases II y III del desarrollo de medicamentos. A su vez los tiempos de evaluación son de algunas semanas. Los ECC pragmáticos tienen como objetivo evaluar la efectividad de las intervenciones, se focalizan más en la medida del síntoma o marcador y maximizan la aplicabilidad y generabilidad del resultado. Los grupos son más heterogéneos y en general son utilizados en la fase IV 0 postautorización (Möller, 2011). Algunos ejemplos de ECC pragmáticos en psiquiatría son los estudios, Catie, Cutlass, Star-D y Star-BD. Un porcentaje importante de la evidencia disponible proviene de ECC explicativos con serias limitaciones para extrapolar los resultados a la PCD. Se estima que una tercera parte de los estudios son llevados a cabo por la industria farmacéutica (Ghaemi, 2015). Uno de los aspectos metodológicos a jerarquizar es el denominado sesgo de selección. Los criterios de inclusión (selección de pacientes) en estos estudios son muy rígidos, esto brinda validez interna al estudio, es decir los datos obtenidos en el mismo metodológicamente son robustos. Sin embargo, quita validez externa, esto significa que la extrapolación de esos datos al resto del universo poblacional es difícil de establecer. Los estudios psicofarmacológicos solo representan un $10 \%$ de los pacientes psiquiátricos (Patsopoulos, 2011). Por otro lado, el sesgo de publicación distorsiona la magnitud del efecto en los meta-análisis, sobreestimando en muchas ocasiones el beneficio observable, esto es más probable en ECC con un número de individuos incluidos bajo. Turner, Matthews, Linardatos, Tell \& Rosenthal (2008) demostraron con los antidepresivos que habría una sobreestimación de la eficacia, debido al sesgo de publicación. 


\section{Entre hedonistas y calvinistas}

Uno de los factores comunes en este tiempo es la ausencia de meta-relatos aglutinadores que permitan brindar unidad conceptual. En este contexto cultural tiene desarrollo y auge la psicofarmacología. Klerman (1972) en un lúcido artículo, destaca la encrucijada en la que estamos. Identifica cuatro cuestiones tanto de índole moral como social: 1 - el "derecho" al tratamiento, 2 - dilemas del tratamiento involuntario en los trastornos mentales por drogas u otras técnicas, 3 - nuevas formas de discapacidad social y cronicidad para pacientes mentales en la comunidad, y 4 controversia sobre si nos estamos convirtiendo o no en sociedades excesivamente medicadas; calvinismo farmacológico y hedonismo psicotrópico. La visión calvinista de la farmacológica involucra una desconfianza general de los medicamentos utilizados para propósitos no terapéuticos y una convicción que si una sustancia "hace sentir bien, debe ser moralmente malo".

La perspectiva del Uso Racional de Medicamento (URM) definido así por la Organización Mundial de la Salud (OMS) como: "Los pacientes reciben la medicación adecuada a sus necesidades clínicas, en las dosis correspondientes a sus requisitos individuales, durante un período de tiempo adecuado y al menor costo posible para ellos y para la comunidad" (2002, p. 1), propone que los medicamentos deben ser usados con un fin terapéutico ya sea sintomático o fisiopatológico y al menor costo posible. Ahora bien, son múltiples las causas por la cual el uso de medicación no cumple con los criterios del uso racional. Para esto inciden factores culturales de los usuarios, características evolutivas de los pacientes portadores de patología psiquiátrica, aspectos vinculados a la medicación y al marketing de las mismas, así como las instituciones de asistencia y los modelos de atención profesional. Es muy común observar en la práctica clínica diaria la demanda de atención psiquiátrica por banalidades de la vida y la necesidad de medicación. A su vez, existe la percepción de un elevado autoconsumo de medicamentos entre los cuales las benzodiacepinas 
encabezan la lista. Es importante considerar que hay disponibilidad en nuestro mercado para acceder a benzodiacepinas sin receta. Como ejemplo la venta ilegal de estos fármacos que en muchas ocasiones se encuentran en ferias barriales, desconociendo su procedencia y condiciones farmacéuticas de almacenamiento. Todo esto en cierta medida nos habla de una cultura de la medicalización que está instalada no solo en nuestro país sino que es de escala global. ¿Estamos en una cultura hedonista que no tolera la angustia? La angustia es inherente a la condición humana y aglutinar toda angustia en un trastorno como plausible acto fármaco-terapéutico genera sobrecarga asistencial y consumo de medicamentos con las consecuencias de aumento del riesgo de reacciones adversas de forma innecesaria, pero muchas veces es difícil de visualizar en el mundo actual. Por otro lado, los hábitos prescriptivos de los psiquiatras no son uniformes. Depende de la zona geográfica y de la formación. Mundt et al. (2012) encontraron prescripciones muy disímiles entre cuatro ciudades alemanas y Kazajistán. Se ha reportado mayor número de prescripciones en hospitales no universitarios y con menos participación en la investigación. Los nuevos constructos nosográficos, como por ejemplo el trastorno por déficit atencional, de elevada prevalencia y muy discutida entidad clínica incrementa el uso de psicotrópicos, así como los usos fuera de indicación (off label) y la prolongación en el tiempo del uso de los medicamentos. Mars et al. (2017) observaron que el aumento en la prescripción de antidepresivos no se debe a un mayor número de personas que comienza con la medicación, sino que parece ser explicada por un aumento en la duración del tratamiento. A su vez, una formación que no sea independiente tiende a aumentar el uso de la medicación. Según Correll y Gallego (2012) habría una asociación para el uso de polifarmacia en aquellos médicos que no asisten a los cursos de educación médica continua y que concurren a programas de educación financiados por la industria farmacéutica. Estos cursos son parte de las estrategias de marketing de la industria a través de la evidencia. Esta es una forma de captar la atención de los 
profesionales, de esta manera se genera como dice Healy (2016) medicina sesgada de la evidencia en vez de medicina basada en la evidencia (en inglés es más elegante ya que sustituye based por biased).

Más aquí o más allá del calvinismo o del hedonismo farmacológico, se debería retomar por una senda crítica, donde predominen hipótesis plausibles, acordes con la clínica y sujeto a derechos de los usuarios, e independencia académica.

\section{La necesidad de un modelo psicopatológico para un adecuado abordaje} terapéutico

En este panorama complejo en el que la cultura incide de manera fundamental en el uso de los psicofármacos y en el que el campo de la psiquiatría se ha expandido, aun en él y especialmente en este, resulta necesario manejar una adecuada hipótesis psicopatológica de enfermedad mental que pueda ordenar dicho campo. En particular dicha hipótesis debiera cumplir una función propedéutica fundamental en relación a la terapéutica.

Encontramos que las hipótesis del órgano-dinamismo de H. Ey (2008) siguen constituyendo una de las principales hipótesis psicopatológicas. Su referencia se motiva en su gran poder heurístico para la identificación y el ordenamiento clínico. A modo sintético puede decirse que en este marco teórico se considera entonces que la enfermedad mental es "enfermedad" que está determinada por un proceso mórbido orgánico, que depende de la patología general, y "mental" en la medida que representa algún tipo de regresión de la vida psíquica. En su expresión, la regresión de la vida psíquica, su estructura es compleja: comprende un aspecto negativo, ausencia y alteración de las funciones psíquicas superiores, y un aspecto positivo, organización nueva de la vida psíquica subsistente. Entre el proceso orgánico generador y el cuadro clínico que es su efecto, se interpone un trabajo psíquico; esto es lo que corresponde a un hiato órgano- clínico. Este "hiato" interpone una distancia que hace problemática 
la reducción de los fenómenos psicopatológicos a los términos somáticos, y que determina que la patología mental, desde esta perspectiva, desobedezca el concepto nosográfico de enfermedad (constituyéndose entonces la patología mental como antinosográfica). Las formas patológicas que corresponden a la psiquiatría representan una disolución global de la función psíquica y representan una regresión de la conciencia o de la personalidad (Ey, 2008).

Los alcances de este trabajo impiden desarrollar las ideas que subyacen a esta noción, pero es necesario hacer alguna puntualización que dé sentido a su pretensión abarcativa. La noción de EM se desarrolla histórica, conceptual y epistemológicamente de manera tal que se asienta firmemente en las formas clínicas que constituyen su centralidad y se va debilitando hacia sus márgenes (esta noción fue desarrollada en conversaciones con $\mathrm{H}$. Casarotti). Sin que esto pretenda asentarse en una noción dimensional de la patología psiquiátrica, pues consideramos que el fenómeno de la EM implica una heterogeneidad y una discontinuidad con respecto a la salud, por otra parte las fronteras que delimitan el concepto se acercan mejor al concepto de margen que al de límite. Desde la centralidad de la noción de EM, la somaticidad del proceso generador y el carácter deficitario estructural del funcionamiento psíquico se va perdiendo en la medida que nos acercamos a la salud, así como el hiato órganoclínico y la globalidad de la afectación psíquica se va perdiendo en cuanto nos acercamos a las enfermedades neuro-psiquiátricas.

De particular utilidad en el discernimiento de los cuadros clínicos resulta la distinción genérica entre enfermedades agudas y enfermedades crónicas. Superando la psicología de las funciones se propone una psicopatología globalista en la que el cuerpo psíquico se organiza, por un lado, en una "estructura" que en un corte transversal de la vida psíquica en cada momento organiza la experiencia actual (campo de la conciencia), y por otro, un corte longitudinal de la persona que constituye el eje de sus modalidades permanentes de adaptación a los acontecimientos de la 
existencia (la personalidad o el ser consciente de sí mismo) (Ey, Bernard \& Brisset, 1978). Personalidad y conciencia constituyen entonces dos "estructuras" no enteramente yuxtapuestas ni enteramente trascendentales cuya alteración patológica diferencial determina las enfermedades mentales crónicas y agudas, respectivamente. La profundización de este esquema es el que permite luego identificar las distintas formas de desestructuración de conciencia (episodios agudos) así como los distintos tipos de estructura psicopatológica alterada en las distintas enfermedades crónicas.

Este esquema, sin pretender constituir una ley absoluta, rígida, de límites netos y sencillos, constituye por otra parte un esquema general de gran utilidad clínica y de gran poder para un adecuado discernimiento sobre las acciones terapéuticas (qué "tratar", cómo "tratar", y qué esperar de los "tratamientos"). Las nociones implícitas en este esquema, por otro lado, no se encuentran presentes en las categorías nosográficas de los "trastornos" del DSM y la CIE, y por lo tanto, no se encuentran representadas en la metodología de evaluación terapéutica de las "evidencias" propia de la MBE o PBE.

\section{Referencias}

Agencia Española de Medicamentos y Productos Sanitarios. (2007) Utilización de antipsicóticos en España (1992-2006). Recuperado de http://www.aemps.gob. es/medicamentosUsoHumano/observatorio/docs/antipsicoticos.pdf

Agencia Española de Medicamentos y Productos Sanitarios. (2015) Informe de utilización de medicamentos. Utilización de medicamentos antidepresivos en España durante el periodo 2000-2013. Recuperado de https://www.aemps.gob.es/medicamentosUsoHumano/observatorio/docs/antide presivos-2000-2013.pdf

Andreasen, N. C. (2007). DSM and the Death of Phenomenology in America: An Example of Unintended Consequences. Schizophrenia Bulletin, 33(1), 108-112. 
Asociación de Psiquiatría Americana. (2002). Manual diagnóstico y estadístico de los trastornos mentales, DSM-IV-TR. Barcelona: Masson.

Baños, J. E., \& Farré, M. (2002). Principios de Farmacología Clínica: bases científicas de la utilización de medicamentos. Barcelona: Masson.

Barcia Salorio, D. (2012), Historia de la Psiquiatría. En Vallejo Ruiloba, J. y Leal Cercós, C. (Eds.), Tratado de Psiquiatría. Madrid: Marbán.

Berrios, G. E. (2008). Historia de los síntomas de los trastornos mentales. México, D.F.: Fondo de Cultura Económica.

Berrios, G. E. (2011). Hacia una nueva epistemología de la psiquiatría. (1ed). Buenos Aires: Polemos.

Bertolote, J. (2008). Raíces del concepto de salud mental. World Psychiatry (Ed Esp.), $6(2)$.

Boorse, C. (1975). On the distinction between disease and illness. Philosophy and Public Affairs, 5, 49-68.

Correll, C. U., \& Gallego, J. A. (2012). Antipsychotic polypharmacy: a comprehensive evaluation of relevant correlates of a long-standing clinical practice. Psychiatr Clin N Am. 35(3), 661-681.

Cuthbert, B. N. (2014). Translating intermediate phenotypes to psychopathology: the NIMH Research Domain Criteria. Psychophysiology. 51(12), 1205-1206. doi: 10.1111/psyp.12342

Evidence-Based Medicine Working Group. Evidence-based medicine. (1992). A new approach to teaching the practice of medicine. Jama, 268(17), 2420-5.

Ey, H. (2008). Estudio No 4, La posición de la psiquiatría dentro del marco de las ciencias médicas (la noción de "enfermedad mental"). En Estudios Psiquiátricos. (p. 67-82.) Buenos Aires: Polemos. 
Ey, H., Bernard, P., \& Brisset, C.H. (1978). Capítulo 1. Parte III: Organización de la vida psíquica. En H. Ey, P. Bernard \& C.H. Brisset, Tratado de Psiquiatría (pp. 2834). ( $8^{\mathrm{a}}$ ed de la $5^{\mathrm{a}}$ ed francesa). Barcelona: Masson.

Gallego, J. A., Bonetti, J., Zhang, J., Kane, J. M., \& Correll, C. U. (2012). Prevalence and correlates of antipsychotic polypharmacy: a systematic review and metaregression of global and regional trends from the 1970s to 2009. Schizophr Res, 138(1), 18-28.

Ghaemi, S. N. (2015). Estadística y Epidemiología en Salud Mental. Guía clínica. (1a ed.) Santiago: Mediterráneo.

Hálfdánarson, Ó., Zoëga, H., Aagaard, L., Bernardo, M., Brandt. L., Fusté, A.C., Furu, K.,... Bachmann, C.J. (2017). International trends in antipsychotics use: a study in 16 countries, 2005-2014. EurNeuropsychopharmacol, 27(10):1064-1076. doi: 10.1016/j.euroneuro.2017.07.001

Healy, D. (2002). The Creation of Psychopharmacology. London: Harvard University Press.

Healy, D. (2016). Psychiatric Drugs Explained. London: Elsevier.

Ilyas, S., \& Moncrieff, J. (2012). Trends in prescriptions and costs of drugs for mental disorders in England, 1998-2010. The British Journal of Psychiatry. 200, 393398. doi: 10.1192/bjp.bp.111.104257

Kendler, K. S. (2016). The nature of psychiatric disorders. World Psychiatry; 15, 5-12

Kingma, E. (2013). Naturalist accounts of mental disorder, The Oxford Handbook of philosophy and psychiatry, Chapter 25, Oxford University Press.

Klerman, G. L. (1972). Psychotropic Hedonism vs. Pharmacological Calvinism. The Hastings Center Report, 2(4), 1-3.

Lanteri Laura, G. (2000). Ensayo sobre los paradigmas de la psiquiatría moderna. Madrid: Tricastela. 
Levin, S. (2010). Apuntes para un análisis epistemológico de algunos problemas de la psiquiatría contemporánea. Vertex. 21(91).

Mars, B., Heron, J., Kessler, D., Davies, N.M., Martin, R.M., Thomas, K.H., \& Gunnell, D. (2017). Influences on antidepressant prescribing trends in the UK: 19952011. Social Psychiatry and Psychiatric Epidemiology, 52(2), 193-200. doi: $10.1007 / \mathrm{s} 00127-016-1306-4$

Mc Hugh, P. R., \& Slavney, P. R. (1985). Perspectivas de la Psiquiatría. Barcelona: Masson.

Möller, H. J. (2011). Effectiveness studies: advantages and disadvantages. Dialogues Clin Neurosci; 13(2), 199-207.

Morkem, R., Barber, D., Williamson, T., \& Patten, S. B. (2012). A Canadian Primary Care Sentinel Surveillance Network Study Evaluating Antidepressant Prescribing in Canada From 2006 to 2012. CanJPsychiatry; 60(12), 564-570.

Mundt, A. P., Aichberger, M. C., Fakhriddinov, S., Fayzirahmanova, M., Grohmann, R., Heinz, A.,... Ströhle, A. (2012). Prescription patterns of patients diagnosed with schizophrenia in mental hospitals in Tashkent/Uzbekistan and in four German cities. Pharmacoepidemiol Drug Saf; 21, 145-151.

Organización Mundial de la Salud. (2002). Promoción del uso racional de medicamentos: componentes centrales. Perspectivas políticas sobre medicamentos de la OMS, (5). Recuperado de http://apps.who.int/medicinedocs/es/d/Js4874s/

Patsopoulos, N. A. (2011). A pragmatic view on pragmatic trials. Dialogues Clin Neurosci, 13(2), 217-224.

Pringsheim, T., \& Gardner, D. M. (2014). Dispensed prescriptions for quetiapine and other second-generation antipsychotics in Canada from 2005 to 2012: a descriptive study. CMAJ Open, 2(4), 225-232. doi: 10.9778/cmajo.2014000 
Stagnaro, J. C. (2006). Elementos para pensar una psiquiatría dinámica hoy. Psicoanálisis APdeBA, 28(1), 171-187.

Teixeira, V., Imbriago, Y., \& Sarries, E. (2015). Utilización de antidepresivos en pacientes ambulatorios del Hospital Policial. Rev Psiquiatr Urug; 79(1), 39-48.

Turner, E. H., Matthews, A.M., Linardatos, E., Tell, R. A., \& Rosenthal, R. (2008). Selective publication of antidepressant trials and its influence on apparent efficacy. N Engl J Med; 358(3), 252-260.

Vallejo Ruiloba, J. \& Leal Cercós, C. (Eds.). (2012). Tratado de Psiquiatría. Historia de la psiquiatría. Madrid: Marbán.

Wakefield, J. C. (1992). Disorder as harmful dysfunction: a conceptual critique of DSMIII-R's definition of mental disorder. Psychological Review, 99, 232-247.

\section{Formato de citación}

Toledo, M., Almada, R., \& Villaba, L. (2018). Paradigmas psiquiátricos y psicofarmacología. ¿Relevancia del órgano-dinamismo de Henry Ey?. Psicología, Conocimiento y Sociedad, 8(2), 258-281. doi: http://dx.doi.org/10.26864/PCS.v8.n2.12 